Voix et Images

volxetimages

\title{
De l'histoire littéraire aux facultés des lettres
}

\section{Bernard Andrès}

Volume 18, numéro 3 (54), printemps 1993

Littérature, folie, altérité

URI : https://id.erudit.org/iderudit/201061ar

DOI : https://doi.org/10.7202/201061ar

Aller au sommaire du numéro

\section{Éditeur(s)}

Université du Québec à Montréal

\section{ISSN}

0318-9201 (imprimé)

1705-933X (numérique)

Découvrir la revue

\section{Citer cet article}

Andrès, B. (1993). De l'histoire littéraire aux facultés des lettres. Voix et Images, 18(3), 641-648. https://doi.org/10.7202/201061ar d'utilisation que vous pouvez consulter en ligne.

https://apropos.erudit.org/fr/usagers/politique-dutilisation/ 
Recherche

\section{De l'histoire littéraire aux facultés des lettres}

\section{Bernard Andrès, Université du Québec à Montréal}

Comment rendre compte d'une vingtaine de titres en quelques feuillets dans une chronique de la recherche? Quels travaux privilégier, comment en regrouper les orientations et qu'en dire en si peu de lignes? En un mot, comment distinguer cette recension de la rubrique "Essai" de notre revue, qui couvre de façon plus approfondie des monographies et autres volumes jouissant déjà d'une bonne diffusion par l'éditeur? Il ne s'agit pas d'introduire un clivage entre les "grands canons" de la recherche ou de la critique et les "Oubliés" du domaine ici rassemblés pour les besoins de la cause. Je fonderai plutôt ma chronique sur la constatation suivante: si certaines équipes bénéficient de débouchés éditoriaux prestigieux chez des éditeurs privés ou universitaires, de nombreuses publications (et pré-publications) émanent aussi de groupes de recherche subventionnés qui font connâtre leurs travaux dans des collections propres, au tirage limité. Leur diffusion repose alors presque exclusivement sur l'accueil de revues spécialisées comme la nôtre et, notamment, sur des chroniques comme celle-ci. Je m'y efforcerai donc de parler surtout de la recherche en émergence et des publications plus marginales, tout en les situant par rapport aux équipes établies et bien (ou mieux) subventionnées. On trouvera donc ici un inventaire minimalement commenté des travaux en cours, de la modeste pré-publication ${ }^{1}$ à la "brique " du CRELIQ ou des Archives des Lettres canadiennes.

Faute de pouvoir détailler chacune des publications, je m'efforcerai de signaler de l'une à l'autre les grandes orientations des travaux 
actuels, en insistant davantage sur certaines directions de recherche qui m'apparaissent renouveler la réflexion sur le littéraire, comme pour cette livraison: l'épistolaire, le biographique et les études comparées. Avant d'aborder ces trois domaines, il convient de souligner la parution d'outils de référence fort utiles, ainsi que de grandes synthèses en histoire littéraire dont certaines devraient faire ailleurs l'objet de recensions plus approfondies.

C'est le cas du deuxième tome de La Vie littéraire au Québec, sous la direction de Maurice Lemire. Ce volume sous-titré "Le projet national des Canadiens" couvre les années 1806 à 1839. Il expose les conditions dans lesquelles s'affermit le champ littéraire québécois, alors marqué par une double tension ainsi caractérisée par les auteurs:

Une première tension, endogène, le nationalisme, ordonne la vie littéraire du Bas-Canada en ce premier tiers du XIX ${ }^{e}$ siècle. Elle alimente la prose d'idées, mais elle agit aussi sur la production d'imagination. Toutefois cette littérature subit l'action d'une autre tension, exogène, celle du romantisme venu d'Europe, en particulier de France. Les pratiques poétiques et narratives y sont alors particulièrement sensibles (p. 2).

Fidèles à l'approche institutionnelle mise en place dans le premier volume, les auteurs présentent d'abord le personnel littéraire (individus et regroupements œuvrant dans le domaine), pour insister ensuite sur des phénomènes marquants comme l'essor de la lecture, de la prose d'idée et des "textes de l'imagination et de la subjectivité . Les synthèses reposent sur une documentation abondante et sont utilement ponctuées de chapitre en chapitre par une section bibliographique intitulée "Pour en savoir davantage*, qui permet au lecteur d'approfondir au besoin la question ${ }^{2}$. Une chronologie détaillée, une abondante bibliographie et de précieux index font de ce volume la référence obligée pour cette période.

À propos de bibliographies, je signale (toujours sous la houlette du Centre de recherche en littérature québécoise de l'Université Laval), la Bibliographie analytique de la science-fiction et du fantastique québécois (1960-1985), sous la direction d'Aurélien Boivin, de Maurice Émond et de Michel Lord ${ }^{3}$. François Ricard, pour sa part, a publié l'Inventaire des archives personnelles de Gabrielle Roy conservées à la Bibliothèque nationale du Canada ${ }^{4}$. Son livre comporte également une liste des autres dépôts d'archives publics concernant l'auteure. On trouvera enfin une bibliographie commentée sur le thème "Ville et littérature. dans la publication Paragraphes du Département d'études françaises de l'Université de Montréal, collection dirigée par Stéphane Vachon. Yves Jubinville et Fabien Ménard situent leur travail dans l'orbe du groupe de recherche «Montréal imaginaire", dirigé par Gilles 
Marcotte et Pierre Nepveu (à qui l'on doit le volume collectif du même nom paru l'an passé chez Fides) ${ }^{\text {s. }}$.

Les actes de colloque donnent aussi lieu à des publications intéressantes, comme celle des éditions du Nordir (du Collège universitaire de Hearst) consacrée aux Frontières et Manipulations génériques dans la littérature canadienne francophone ${ }^{6}$. Il s'agit là d'une rencontre au département d'études françaises de l'Université d'Ottawa, en mai 1992. Des étudiants venus de plusieurs institutions canadiennes s'intéressent à la question du décloisonnement et du renouvellement des genres. Les aspects historiques et théoriques de la problématique voisinent avec des cas de figure illustrés par les œuvres d'Hubert Aquin, VictorLévy Beaulieu, Jacques Brault, Anne Hébert et Jacques Ferron. La conférencière invitée, Ginette Michaud, propose pour finir un brillant état de la question avec ses "Quelques réflexions sur les transferts génériques. Plus éclectique mais non moins digne d'intérêt, Le Roman québécois depuis 1960. Métbodes et analyses ${ }^{7}$ témoigne d'un colloque international aux Pays-bas, où les chercheurs avaient relevé l'étrange défi: "présenter, dans un premier temps, une approche méthodologique donnée, puis l'appliquer dans une analyse concrète d'un roman québécois publié après 1960* (p. 7). Psychanalyse, psychocritique, thématique, rhétorique, stylistique, axiologie, dialogisme, sémiotique et narratologie, déconstruction, lecture au féminin, histoire littéraire et sociocritique "s'appliquent " donc à des corpus aussi divers que ceux d'Anne Hébert, Marie Josée Thériault, Jacques Godbout, Jacques Brault, Régine Robin, Jacques Poulin, Gabrielle Roy, Nicole Brossard, Hubert Aquin ou Gérard Bessette. Tels auteurs résistent mieux que d'autres à telles grilles et vice-versa. Outre quelques interventions plus inspirées (qui déjouent heureusement la dichotomie programmée), l'entreprise offre un certain intérêt pédagogique: l'étudiant y trouvera une sélection d'approches et de méthodologies bien synthétisées et utilement agrémentées d'« exemples" et d'*applications". Certains articles proposent aussi des synthèses plus en rapport avec le titre de l'ouvrage qu'avec son sous-titre: "Stylistique et recul des genres au Québec", de Madeleine Frédéric, «Fragments de journaux intimes dans le discours du roman", de Pierre Hébert, "L'inscription d'un héritage littéraire québécois dans le roman des années quatre-vingt", de Lucie Robert, "L'édition du roman québécois, 1961-1974", de Jacques Michon, ou "La bibliométrie: le cas des best-sellers", de Denis Saint-Jacques. Il faut aussi signaler dans ce dernier domaine l'ouvrage de Denis SaintJacques et Roger de la Garde (directeurs), Les Pratiques culturelles de grande consommation. Le marché francopbone ${ }^{8}$. Les auteurs y font une mise au point non dépourvue de polémique sur les questions de 
cultures "savante ", "élargie ", " de masse ", "industrielle ", "médiatique" et " de grande consommation":

Notre francophonie peut bien être brillamment illustrée par Réjean Ducharme, Gaston Miron ou Nicole Brossard, elle se réalise de façon tout aussi nécessaire et peut-être même plus par Bernard Derome, Croc, Roch Voisine ou Arlette Cousture (p. 13).

C'est tout le problème d'une définition du "culturel * assez large pour ne pas s'en tenir au champ restreint des lettres ou des arts, mais suffisamment circonscrite pour ne pas englober la multitude des manifestations sociologiques et anthropologiques de l'activité humaine. Josette Féral, on s'en souvient, s'en était inquiétée dans son essai d'économie politique du théâtre 9 . La réflexion fut récemment reprise par Joseph Melançon et ses collaborateurs qui ont entrepris d'examiner la culture comme " métaphore de notre vue, de notre point de vue ${ }^{10}$.

Pour revenir aux synthèses, mentionnons la parution du Roman contemporain au Québec (1960-1985), sous la direction de François Gallays, Sylvain Simard et Robert Vigneault ${ }^{11}$. Ce tome VIII des Archives des Lettres canadiennes prend le relais du tome III de 1964 (alors consacré au Roman canadien-français qui avait connu deux rééditions, une en 1971 et l'autre en 1977). Jacques Allard y présente les romans des années cinquante marqués au coin du "réalisme spirituel", alors que Gilles Marcotte retrace la modernité des œuvres majeures de 1960 à 1985: Ferron, Thériault, Aquin, Bessette, Godbout, Basile, Ducharme, Blais, etc. François Gallays, pour sa part, aborde les "jeunes" auteurs contemporains: Lalonde, Noël, Mistral, Brulotte, Larue, Poulin, Turgeon, Savoie, etc. Le reste des études porte sur des auteurs particuliers dont l'œuvre entière (ou telle production) se trouve relue et analysée sur le mode monographique. Aquin, Hébert et Maillet, bien sûr, mais aussi Archambault, Beauchemin, Beaulieu, Brossard, Caron, Carrier, Major, Villemaire et Tremblay, notamment. John Hare signe enfin une bibliographie chronologique de tous ces romans, de 1970 à 1985.

J'annonçais plus haut certaines orientations de recherche qui semblent renouveler la réflexion sur le littéraire. Les études comparées en font partie. Qu'elles touchent de près ou de loin au corpus québécois, elles permettent au lecteur de déporter son point de vue et de percevoir sous un autre angle les productions d'ici... et d'ailleurs. C'est le cas des Confluences littéraires ${ }^{12}$ de Zila Bernd et Michel Peterson (directeurs) qui examinent "les bases d'une comparaison" entre le Brésil et le Québec. Une quinzaine de chercheurs des deux pays littéraires entrecroisent leurs études du tertium comparationis (W. Godzich), du "nouveau monde" (C. Gamache), de "l'inclusion de 
la parole de l'autre" (Z. Bernd), de l'aanthropophagie culturelle" (W. Moser, L. Perrone-Moises), des questions de filiation (S. Harel) et, bien sûr, d'“américanité. (M. Laroche ${ }^{13}$ ). D'autres études comparatives portent sur $\mathrm{H}$. Aquin et $\mathrm{O}$. Lins, A. Dourado et J. Godbout, N. Pinon, L. Fagundes Telles et A. Hébert, ou sur les corpus poétiques du Québec et du Brésil. On trouvera parmi les poèmes en traduction qui ouvrent le recueil d'articles une superbe "Marcha ao amor " de Gaston Miron (traduction de Flavio Aguiar). L'état présent de la recherche dans le domaine est signé par Lilian Pestre de Almeida, Zila Bernd et votre serviteur. Chez le même éditeur, paraissaient récemment les actes d'un colloque de 1991 sur L' Indien", instance discursive ${ }^{14}$. Il s'agissait, dans le sillage de l'“année Colomb", d'étudier " le regard occidental sur l'Amérindien.. L'optique choisie est celle de l'analyse discursive pratiquée dans le groupe de recherche MARGES (Marginalisation et marginalité dans les pratiques discursives) et au CIADEST (Centre interuniversitaire d'analyse du discours et de sociocritique des textes), à Montréal. Aux études sur Colomb (Gómez-Moriana), l'Inca Garcilaso (C. Poupeney Hart), Lope de Vega (T. J. Kirschner) et l'abbé Raynal (M. Duchet), répondent les travaux sur Cartier, Champlain et Lejeune (R. Ouellet), Lafitau (A. Motsch), ou sur le discours indigéniste dans les littératures latino-américaines.

Les travaux sur le biographique et l'épistolaire m'apparaissent occuper une place nouvelle dans le champ de la recherche, qu'il s'agisse de l'édition critique de correspondances, de biographies intellectuelles ou de réflexion théorique sur "Les facultés des lettres". C'est là le titre donné par Benoît Melançon et Pierre Popovic aux études qu'ils viennent de rassembler sur l'épistolaire français et québécois, objet d'un colloque tenu lors du dernier Congrès de l'ACFAS à Montréal ${ }^{15}$. Dans leur présentation de l'ouvrage, les éditeurs soulignent l'importance croissante accordée à ces recherches depuis une dizaine d'années. C'est à l'occasion de la décade de Cerisy-la-Salle en 1987, consacrée à l'épistolarité à travers les siècles, que naquit l'Association interdisciplinaire de recherche sur l'épistolaire (AIRE). Étudier la lettre, expliquent Melançon et Popovic, donne

\footnotetext{
l'impression, et peut-être l'illusion, de rejoindre un arrière-lieu des Lettres, c'est-à-dire un espace retiré de la feinte où du sujet se donne déjà à lire, autrement (mais ni plus ni moins authentiquement) que dans les textes figés par la publication. Il vivote là du littéraire avant la lettre, si l'on peut dire, vivant parce que nécessairement inachevé, parce qu'en attente d'une réponse ${ }^{16}$.
}

Une dizaine de chercheurs s'interroge donc ici sur cet espace infralittéraire différemment occupé par Diderot (Benoît Melançon), 
Crémazie et Casgrain (Micheline Cambron), Rimbaud (Pierre Popovic), Buies (Francis Parmentier), Camille Roy (Jane Everett), Alfred DesRochers (Richard Giguère), Saint-Denys Garneau (Joseph Bonenfant), Grandbois (Bernard Chassé),-Élie et Borduas-(Gilles Lapointe),-Miron et Haeffely (Louise Dupré). Manon Brunet qui prépare, on le sait, l'édition de la correspondance de Casgrain, étudie ici *Les traités d'art épistolaire au XIXe siècle québécois", dont le modèle reste le Court Traité sur l'art épistolaire de Jean-Baptiste Meilleur (1845). Un récent colloque tenu à l'Université York vient aussi d'aborder la question des manuels de correspondance, ainsi que, de façon plus générale "La lettre au XVnI ${ }^{e}$ siècle et ses avatars ${ }^{17}$. Dans le champ "québécois", Réal Ouellet y a traité de l'épistolarité dans les relations de voyage, Jack Warwick, de Lafitau, William Eccles, de la correspondance entre la Nouvelle-France et le Ministère de la Marine à Versailles, Benoît Melançon de la correspondance de Bougainville au Canada, Martin Robitaille et Nicole Deschamps d'Élizabeth Bégon, et votre serviteur des fausses Lettres du Marquis de Montcalm.

L'examen de la correspondance des hommes de lettres ne manque pas de compléter utilement l'étude de leurs œuvres. Elle est souvent à la base de leur biographie littéraire. En témoignent l'opuscule de Jacques Blais, Hélène Marcotte et Roger Saumur, Louis Fréchette épistolier ${ }^{18}$, ou la magistrale édition de la Correspondance d'Artbur Buies par Francis Parmentier ${ }^{19}$. C'est en puisant notamment dans la correspondance retrouvée de Charles ab der Halden, que MarieAndrée Beaudet a pu reconstituer la carrière du critique français qui défendait au début du siècle, contre Jules Fournier, que la littérature canadienne-française existait bien ${ }^{20}$. Après avoir finement retracé la biographie intellectuelle d'ab der Halden, Marie-André Beaudet reproduit le texte de la querelle historique, ainsi qu'une sélection de lettres du et au critique. De son côté, Roger Le Moine avait travaillé sur un fonds de correspondances inédites pour présenter l'histoire des deux premières loges montréalaises du Grand Orient de France: il s'agissait de correspondances (secrètes) des francs-maçons québécois de "L'Émancipation" et de *Force et courage*, entre 1896 et $1924^{21}$. De tels travaux patiemment entrepris dans les fonds d'archives permettent de mieux comprendre l'histoire des idées à travers la carrière de certains individus et, comme le dit justement Roger Le Moine, de songer à *un ouvrage d'ensemble sur la contestation au Québec depuis la montée de l'ultramontanisme * (p. XI). Louis-Joseph Papineau, Honoré Mercier, Honoré Beaugrand, Arsène Bessette et bien d'autres avant et après eux ont alimenté la pensée libérale au Québec, en dépit des persécutions dont ils faisaient l'objet (qu'on songe notam- 
ment, dans les années 1910 au Débutant de Bessette et au climat dans lequel fut "reçue "La Scouine de Laberge). L'histoire de ces courants de pensée reste à faire. Nous aurons l'occasion d'y revenir dans d'autres chroniques.

1. Signalons à ce chapitre les Cahiers du GREL (Groupe de recherche sur la lecture), au Département d'études littéraires de l'Université du Québec à Montréal. Le numéro 7 de janvier 1993 (signé Rachel Bouvet et Bertrand Gervais) s'intitule De la dissimulation à l'alphabétisation: les nouveaux lecteurs (28 p.). Le numéro 8 , de février 1993, signé Jean Valenti, porte sur Ducharme: Les Mille Milles et une surprise du lecteur: l'exemple du Nez qui voque ( 90 p.). Une nouvelle collection vient aussi de paraître au même département de l'UQAM: les Cahiers de l'ALAQ (Archéologie du littéraire au Québec). Ce groupe de recherche dont je m'occupe depuis deux ans étudie les premières manifestations du littéraire au tournant du $\mathrm{XIX}^{\mathrm{C}}$ siècle (1764-1815). La première livraison, signée Bernard Andrès et Pascal Riendeau, s'intitule La Conquête des Lettres au Québec (1764-1815): florilège, (Montréal, UQAM, Département d'Études littéraires, Cahiers de l'ALAQ, numéro 1, mars 1993, 446 p.). Il s'agit d'une compilation des textes - fondateurs ?, de Bailly de Messein à Pierre de Sales Laterrière, en passant par Ross Cuthbert, Pierre du Calvet, Valentin Jautard, Henry-Antoine Mézière, Joseph Quesnel, Pierre Roubaud et quelques autres.

2. Je ne peux ici commenter certains points de détail ou d'interprétation sur lesquels je reviendrai dans les cahiers de l'ALAQ.

3. Aurélien Boivin, Maurice Émond et Michel Lord (directeurs), Bibliographie analytique de la science fíction et du fantastique québécois (1960-1985), Québec, Nuit blanche éditeur, 1992, $584 \mathrm{p}$.

4. François Ricard, Inventaire des archives personnelles de Gabrielle Roy conservées à la Bibliothèque nationale du Canada, Montréal, Boréal, 1992, 204 p. Toujours désireux de compléter l'inventaire, François Ricard invite les personnes et institutions possédant des documents de Gabrielle Roy à le lui faire savoir.

5. Gilles Marcotte et Pierre Nepveu, Montréal imaginaire: Ville et litterature, Montréal, Fides, 426 p. Pour la bibliographie de Yves Jubinville et Fabien Ménard, voir Paragraphes, Département d'études françaises de l'Université de Montréal, coll. - Ville et littérature: Bibliographie commentée •, no 7, 1992, 140 p.

6. En collaboration, Frontières et manipulations génériques dans la littérature canadienne francophone, Hearst, Éditions du Nordir, 1992, 138p. Lucie Lalonde et Pierre Siguret co-organisaient ce colloque étudiant. Sur la notion de genre littéraire, on consultera avec profit les actes d'un colloque tenu en 1988 à l'Université de l'Alberta, publiés voici deux ans par Claudine Potvin et I. S. MacLaren: Literary Genres/Les Genres littéraires, Edmonton, University of Alberta, Research Institute for Comparative Literature, 1991, 164 p. Dans un autre domaine, celui de l'édition critique, un autre colloque de 1988 a dû attendre quatre ans pour être publié; il s'agit de l'ouvrage de Paul-André Bourque, Pierre Hétu, Marty Laforest, Vincent Nadeau (directeurs), Editer des ceuvres médiatiques, Québec, Nuit blanche éditeur, coll. . Les Cahiers du CRELIQ •, 1992, 148 p.

7. Louise Milot et Jaap Lintvelt (directeurs), Le Roman québécois depuis 1960. Méthodes et analyses, Sainte-Foy, Les Presses de l'Université Laval, Centre de recherche en littérature québécoise, 1992, 320 p. Actes d'un colloque organisé par le Centre d'études canadiennes de l'Université de Groningue et le CRELIQ de l'Université Laval.

8. Denis Saint-Jacques' et Roger de la Garde (directeurs), Les Pratiques culturelles de grande consommation. Le marché francophone, Québec, Nuit blanche éditeur, coll. .CEFAN, 1992, 259 p. 
9. Josette Féral, La Culture contre l'art. Essai d'économie politique du théâtre, Sillery, Presses de l'Université du Québec, 1990 (voir surtout le premier chapitre).

10. Joseph Melançon (directeur), Les Métaphores de la culture, Sainte-Foy, Presses de l'Université Laval, CEFAN, 296 p. (voir notamment pour la littérature les articles de Guy Bouchard, :La métaphore androcentrique de la culture, de Pierre Ouellet, - Le changement de lieux. Culture et métaphore., d'Yves Préfontaine, : La métaphorisation des origines: une quête limite et de Joseph Melançon, *La littérature comme capital sémiotique .).

11. François Gallays, Sylvain Simard et Robert Vignault (directeurs), Le Roman contemporain au Québec, 1960-1985, Montréal, Fides, coll. Archives des lettres canadiennes ,, t. 8, 1992, 551 p.

12. Zila Bernd et Michel Peterson, Confluences littéraires. Brêsil-Québec: les bases d'une comparaison, Candiac, Les Éditions Balzac, 1992, 376 p.

13. De Maximilien Laroche, il faut surtout saluer la récente parution de l'essai Dialectique de l'américanisation, Sainte-Foy, Université Laval, Département des littératures, GRELCA, collection - Essais ', n 10, 1993, 314 p. (le premier chapitre porte sur le Québec, notamment la poésie de Roland Giguère). Maximilien Laroche a déjà publié dans la même collection du GRELCA un certain nombre d'essais sur les littératures francophones d'Afrique et d'Amérique (surtout de la Caraïbe), notamment une étude pénétrante sur La Découverte de l'Amérique par les Américains (1989).

14. Antonio Gómez-Moriana et Danielle Trottier, L's'Indien ", instance discursive. Actes du Colloque de Montréal 1991, Candiac, Les Éditions Balzac, 462 p.

15. Benoît Melançon et Pierre Popovic, La Faculté des lettres. Recherches récentes sur l'épistolaire français et québécois, Montréal, Centre universitaire pour la sociopoétique de l'épistolaire et des correspondances, Département d'études françaises, Université de Montréal, février 1993, 241 p. (actes du congrès organisé par le Centre universitaire pour la sociopoétique de l'épistolaire et des correspondances, à l'Université de Montréal en mai 1992).

16. Ibid., p. 5-6.

17. Colloque international à l'Université York, du 29 avril au 1er mai 1993, organisé par Georges Bérubé et Marie-France Silver (actes à paraître).

18. Jacques Blais, Hélène Marcotte et Roger Saumur, Louis Fréchette épistolier, Québec, Nuit blanche éditeur, 1992, 84 p.

19. Arthur Buies, Correspondance (1855-1901), édition préparée, présentée et annotée par Francis Parmentier, Montréal, Guérin Littérature, 1993. On doit aussi à cet infatigable chercheur la fameuse édition critique des Chroniques d'Arthur Buies: Cbroniques I, et Chroniques II, Montréal, Les Presses de l'Université de Montréal, 1986, 657p. et 1991, 504 p.

20. Sur cette période du tournant du $\mathrm{xx}^{e}$ siècle et sur le climat idéologique de l'époque, $c f$. Marie-Andrée Beaudet, Charles ab der Halden. Portrait d'un inconnu, Montréal, l'Hexagone, $235 \mathrm{p}$.

21. Roger Le Moine, Deux Loges montréalaises du Grand Orient de France, Ottawa, Les Presses de l'Université d'Ottawa, 1991, $190 \mathrm{p}$. 